\title{
Solving Stochastic AC Power Flow via Polynomial Chaos Expansion
}

\author{
Tillmann Mühlpfordt, Timm Faulwasser ${ }^{a}$, Veit Hagenmeyer
}

\begin{abstract}
The present contribution demonstrates the applicability of polynomial chaos expansion to stochastic (optimal) $\mathrm{AC}$ power flow problems that arise in the operation of power grids. For rectangular power flow, polynomial chaos expansion together with Galerkin projection yields a deterministic reformulation of the stochastic power flow problem that is solved numerically in a single run. From its solution, approximations of the true posterior probability density functions are obtained. The presented approach does not require linearization. Furthermore, the IEEE 14 bus serves as an example to demonstrate that the proposed approach yields accurate approximations to the probability density functions for low orders of polynomial bases, and that it is computationally more efficient than Monte Carlo sampling.
\end{abstract}

Index Terms - optimal power flow, stochastic uncertainty, polynomial chaos expansion

\section{INTRODUCTION}

The solution of the power flow equations is of paramount importance for the design, operation, and control of electrical networks [1], [2]. The objective of the so-called power flow problem is to find operating conditions for a set of connected power generating units given load specifications. ${ }^{1}$ Usually, uncertainties and model-plant mismatch are ignored, leading to deterministic power flow problems. The main challenges are then the development of distributed computational algorithms [3] and the construction of suitable convex relaxations [4]. However, it is well-known that power flow equations are subject to uncertainty. Typical sources of uncertainty in power flow problems are generation or load profiles, line parameters, and/or network conditions [5]. With increasing penetration of renewable energy sources-which are uncertain due to their volatility - a structured approach to solve uncertain power flow problems is desirable, especially for later use in context of power dispatch, cf. [6].

When uncertainties in the power network are treated as random variables, the problem is referred to as stochastic power flow or probabilistic power flow. Early results on decoupled stochastic power flow have been presented in [7]. So-called intrusive convolution techniques are discussed in [8], [9], while Fast Fourier Transformations are investigated

All authors are with the Institute for Applied Computer Science, Karlsruhe Institute of Technology, 76344 Eggenstein-Leopoldshafen, Germany \{tillmann.muehlpfordt, timm.faulwasser, veit.hagenmeyer\}akit.edu.

This work was supported by the Helmholtz Association under the Join Initiative "Energy System 2050 - A Contribution of the Research Field Energy".

${ }^{a} \mathrm{TF}$ is also with the Laboratoire d'Automatique, École Polytechnique Fédérale de Lausanne, CH-1015 Lausanne, Switzerland. TF is indebted to the Baden-Württemberg Stiftung for the financial support of this research project by the Elite Programme for Postdocs.

${ }^{1}$ The term load flow is used interchangeably in the literature. in [10]. Gram-Charlier Expansions are used in [11], whose main advantage is computational efficiency, i.e. the method allows to approximate the posterior probability distributions with a single run. The mentioned analytical methods are limited in the sense that they can tackle either linearized AC power flow or (intrinsically linear) DC power flow.

In contrast to the analytical approaches, so-called nonintrusive sampling-based methods allow consideration of nonlinearities. Monte Carlo methods require to solve the nominal problem for a large number of samples drawn from a set with a specified probability distribution, see [12] for a general introduction and [13] for results on stochastic power flow. Point Estimate Methods generate approximations to true probability distributions by matching the first few stochastic moments based on a small number of samples [14], [15], [16]. Typically, the number of simulations is twice the number of uncertain variables. The application of Point Estimate Methods to stochastic power flow, and their outperformance of Monte Carlo methods, are demonstrated in [17], [18] for networks with up to 129 buses. Finally, Unscented Transforms, which are well-known from their application to Kalman Filtering [19], can be applied to stochastic power flow problems, see [20], [21]. The number of required samples is only marginally larger compared to Point Estimate Methods, yet the results using Unscented Transforms are more accurate. This is demonstrated via simulations for a 574 bus network in [20].

The present contribution discusses the application of Polynomial Chaos Expansion (PCE) to solve the stochastic (optimal) AC power flow problem numerically in a single run. This intrusive uncertainty propagation technique relies on spectral expansion of all random variables to reformulate the stochastic problem as a structurally equivalent deterministic problem of larger dimension [12]. In contrast to sampling-based techniques for which a large number of deterministic problems is solved, the enlarged deterministic problem stemming from PCE has to be solved only once, i.e. in a single numerical run. Sampling-based techniques create a large amount of data that has to be handled. PCE reduces the necessary data to the PCE coefficients, from which the posterior distributions can be sampled efficiently, and stochastic moments can be computed easily. Compared to convolution-based techniques, PCE allows to consider polynomial nonlinearities. PCE exhibits fast convergence if the random variables enter the problem smoothly [12]. Originating in works of Norbert Wiener in the 1930s, PCE has gained attention in the control community recently, especially in combination with model predictive control [22], [23], [24]. In the present contribution, we show that PCE offers 
a structured approach to explicitly account for uncertainties via probability density functions in AC power flow problems.

Furthermore, PCE is not restricted to Gaussian random variables. Instead, it can cope with arbitrary random variables (with finite variance). This is of interest for power flow problems, because aggregated load profiles do not exhibit Gaussian distributions, in general. Instead, Beta/Gamma/lognormal distributions yield more accurate results [25].

The remainder of the work is structured as follows: Section II recalls the deterministic $\mathrm{AC}$ power flow problem. In Section III we introduce a PCE formulation for stochastic AC power flow problems, which is extended to stochastic optimal AC power flow in Section III-B. We draw upon the IEEE 14 bus example to demonstrate the applicability of PCE to stochastic AC power flow problems in Section IV.

\section{Deterministic AC Power Flow}

Consider an electrical AC network composed of $N \in \mathbb{N}$ buses (or nodes), where $\mathcal{N}=\{1, \ldots, N\}$ is the bus index set. Let the entries of the bus admittance matrix $\vec{Y} \in \mathbb{C}^{N \times N}$ be given in rectangular representation $\vec{y}_{i j}=g_{i j}+\mathrm{i} b_{i j} \in \mathbb{C}$ for all $i, j \in \mathcal{N}$, where $\mathrm{i}$ is the imaginary unit. The power phasor $\vec{s}_{i} \in \mathbb{C}$ and voltage phasor $\vec{v}_{i} \in \mathbb{C}$ fully describe the state of each bus $i \in \mathcal{N}$. Based on the voltage phasor representation, we distinguish between polar and rectangular formulations,

$$
\begin{array}{lll}
\text { polar: } & \vec{v}_{i}=v_{i} \mathrm{e}^{\mathrm{i} \delta_{i}}, & \vec{s}_{i}=p_{i}+\mathrm{i} q_{i}, \\
\text { rectangular: } & \vec{v}_{i}=v_{i}^{\mathrm{re}}+\mathrm{i} v_{i}^{\mathrm{im}}, & \vec{s}_{i}=p_{i}+\mathrm{i} q_{i},
\end{array}
$$

where $i \in \mathcal{N}$. Then, the deterministic polar (optimal) power flow problem is defined as

$$
\begin{aligned}
& \underset{x^{p}}{\operatorname{minimize}} \quad J\left(x^{p}\right) \\
& \text { subject to } \forall i \in \mathcal{N} \\
& x_{i}^{p}=\left[\begin{array}{llll}
v_{i} & \delta_{i} & p_{i} & q_{i}
\end{array}\right]^{\top} \in \mathbb{R}^{4}, \\
& p_{i}=\sum_{j \in \mathcal{N}} v_{i} v_{j}\left(g_{i j} \cos \left(\delta_{i}-\delta_{j}\right)+b_{i j} \sin \left(\delta_{i}-\delta_{j}\right)\right), \\
& q_{i}=\sum_{j \in \mathcal{N}} v_{i} v_{j}\left(g_{i j} \sin \left(\delta_{i}-\delta_{j}\right)-b_{i j} \cos \left(\delta_{i}-\delta_{j}\right)\right),
\end{aligned}
$$$$
\text { bus specifications for all buses } i \in \mathcal{N} \text {, }
$$

where the decision variables are $x^{p}=\left[x_{1}^{p^{\top}}, \ldots, x_{N}^{p^{\top}}\right]^{\top} \in$ $\mathbb{R}^{4 N}$. Similarly, the deterministic rectangular (optimal) power flow problem is defined as

$$
\begin{aligned}
& \underset{x}{\operatorname{minimize}} \quad J(x) \\
& \text { subject to } \forall i \in \mathcal{N} \\
& x_{i}=\left[\begin{array}{llll}
v_{i}^{\mathrm{re}} & v_{i}^{\mathrm{im}} & p_{i} & q_{i}
\end{array}\right]^{\top} \in \mathbb{R}^{4}, \\
& p_{i}=\sum_{j \in \mathcal{N}} g_{i j}\left(v_{i}^{\mathrm{re}} v_{j}^{\mathrm{re}}+v_{i}^{\mathrm{im}} v_{j}^{\mathrm{im}}\right)+b_{i j}\left(v_{i}^{\mathrm{im}} v_{j}^{\mathrm{re}}-v_{i}^{\mathrm{re}} v_{j}^{\mathrm{im}}\right), \\
& q_{i}=\sum_{j \in \mathcal{N}} g_{i j}\left(v_{i}^{\mathrm{im}} v_{j}^{\mathrm{re}}-v_{i}^{\mathrm{re}} v_{j}^{\mathrm{im}}\right)-b_{i j}\left(v_{i}^{\mathrm{im}} v_{j}^{\mathrm{im}}+v_{i}^{\mathrm{re}} v_{j}^{\mathrm{re}}\right), \\
& \text { bus specifications for all buses } i \in \mathcal{N},
\end{aligned}
$$

where the decision variables are $x=\left[x_{1}^{\top}, \ldots, x_{N}^{\top}\right]^{\top} \in \mathbb{R}^{4 N}$. The power flow problems (2) and (3) involve not yet defined bus specifications. A bus specification defines two quantities at each bus $i \in \mathcal{N}$. Consequently, if a specification is given for each bus $i \in \mathcal{N}$ in terms of two variables, then the problems (2) and (3) involve $4 N$ unknowns and $4 N$ equations. Thus, they reduce to feasibility problems that are commonly referred to as power flow problems. In this case, the objective $J: \mathbb{R}^{4 N} \rightarrow \mathbb{R}$ can be neglected. If, however, not all buses are subject to a specification, the problems are called optimal power flow problems. In this case, the choice of the cost function $J$ is of particular interest and additional inequality constraints might be considered, e.g. active power generation limits, or transmission limits.

The common bus specifications are, cf. [1], [2],

$$
\begin{array}{r}
\text { Slack: }\left[\begin{array}{llll}
1 & 0 & 0 & 0 \\
0 & 1 & 0 & 0
\end{array}\right] x_{i}^{p} \stackrel{!}{=}\left[\begin{array}{l}
v^{\mathrm{ref}} \\
\delta^{\mathrm{ref}}
\end{array}\right] \Longleftrightarrow C^{\mathrm{sl}} x_{i}^{p}=c_{i}^{\mathrm{sl}}, \\
\text { PQ: }\left[\begin{array}{llll}
0 & 0 & 1 & 0 \\
0 & 0 & 0 & 1
\end{array}\right] x_{i}^{p} \stackrel{!}{=}\left[\begin{array}{l}
p_{i}^{\mathrm{ref}} \\
q_{i}^{\mathrm{ref}}
\end{array}\right] \Longleftrightarrow C^{\mathrm{pq}} x_{i}^{p}=c_{i}^{\mathrm{pq}}, \\
\text { PV: }\left[\begin{array}{llll}
0 & 0 & 1 & 0 \\
1 & 0 & 0 & 0
\end{array}\right] x_{i}^{p} \stackrel{!}{=}\left[\begin{array}{l}
p_{i}^{\mathrm{ref}} \\
v_{i}^{\mathrm{ref}}
\end{array}\right] \Longleftrightarrow C^{\mathrm{pv}} x_{i}^{p}=c_{i}^{\mathrm{pv}} .
\end{array}
$$

Note that they are given in terms of polar power flow state variables $x_{i}^{p}$. Without loss of generality, the reference voltage angle $\delta^{\text {ref }}$ of the slack bus $i_{\mathrm{sl}} \in \mathcal{N}$ is zero. Hence, the specification of the slack bus and the PQ buses is the same for polar and rectangular power flow. However, for rectangular power flow, each PV bus defines a quadratic constraint with respect to the voltage magnitude. In rectangular power flow, the bus specifications are

$$
\begin{aligned}
& \text { Slack: }\left[\begin{array}{cccc}
1 & 0 & 0 & 0 \\
0 & 1 & 0 & 0
\end{array}\right] x_{i} \stackrel{!}{=}\left[\begin{array}{c}
v^{\mathrm{ref}} \\
0
\end{array}\right] \Longleftrightarrow C^{\mathrm{sl}} x_{i}=c_{i}^{\mathrm{sl}}, \\
& \mathrm{PQ}:\left[\begin{array}{llll}
0 & 0 & 1 & 0 \\
0 & 0 & 0 & 1
\end{array}\right] x_{i} \stackrel{!}{=}\left[\begin{array}{c}
p_{i}^{\mathrm{ref}} \\
q_{i}^{\mathrm{ref}}
\end{array}\right] \Longleftrightarrow C^{\mathrm{pq}} x_{i}=c_{i}^{\mathrm{pq}} \text {, }
\end{aligned}
$$

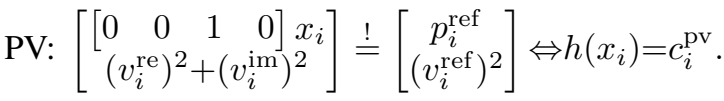

Compared to polar power flow with (2) its trigonometric nonlinearities, rectangular power flow (3) is at most quadratic in the decision variables. This polynomial nonlinearity can be exploited for stochastic (optimal) power flow.

\section{Stochastic AC Power Flow Via PCE}

We speak of the stochastic power flow problem if the bus reference values in (4) are described via probability density functions (PDFs). The mathematical structure of this problem is similar to the deterministic power flow problem: compute PDFs that satisfy a system of nonlinear algebraic equations. ${ }^{2}$ One computationally expensive solution is to run Monte Carlo simulations. In what follows, we will use a different approach based on Polynomial Chaos Expansion (PCE).

\footnotetext{
${ }^{2}$ Arguably, this is a restriction to only uncertain generation and/or uncertain supply. An extension would be to allow for uncertain network structure and/or uncertain line parameters [5]. This, however, is beyond the scope of the present contribution.
} 
TABLE I

ASKEY-SCHEME FOR $2^{\text {nd }}$-ORDER CONTINUOUS RANDOM VARIABLES.

\begin{tabular}{lll} 
Type & Support & Polynomial Basis \\
\hline Beta & $(-1,1)$ & Jacobi \\
Gamma & $(0, \infty)$ & Laguerre \\
Gaussian & $(-\infty, \infty)$ & Hermite \\
Uniform & {$[-1,1]$} & Legendre
\end{tabular}

\section{A. Polynomial Chaos Expansion}

Consider the random vector $\xi=\left[\xi_{1}, \ldots, \xi_{n_{\xi}}\right]^{\top}$ with PDF $\rho(\xi)$ and assume each element is a second-order continuous standard random variable, i.e. $\xi \in \mathrm{L}^{2}\left(\Omega_{\xi}, \mathrm{F}_{\xi}, \mathrm{P}_{\xi}\right)$, and $\xi_{i}$ is Gaussian, uniform, Beta, or Gamma for all $i \in\left\{1, \ldots, n_{\xi}\right\} .{ }^{3}$ Polynomial chaos allows to rewrite general second-order continuous variables $z(\xi)$ as

$$
z(\xi)=\sum_{l=0}^{\infty} z_{l} \phi_{l}(\xi)
$$

where $z_{l} \in \mathbb{R}$ are the deterministic PCE coefficients. The basis functions $\phi_{l}: \Omega_{\xi} \rightarrow \mathbb{R}$ are orthogonal polynomials,

$$
\mathrm{E}\left[\phi_{l}(\xi) \phi_{k}(\xi)\right]=\int_{\Omega_{\xi}} \phi_{l}(\tau) \phi_{k}(\tau) \rho(\tau) \mathrm{d} \tau=\gamma_{l} \delta_{l k},
$$

where $\mathrm{E}[\cdot]$ is the expected-value operator, $\gamma_{l}$ is a positive constant, and $\delta_{l k}$ is the Kronecker-delta.

Remark 1 (Hilbert space $\mathrm{L}^{2}(\cdot)$ ): The space of secondorder random variables $\mathrm{L}^{2}\left(\Omega_{\xi}, \mathrm{F}_{\xi}, \mathrm{P}_{\xi}\right)$ is a Hilbert space [12]. The basis is spanned by the respective polynomials from Table I, and the scalar product is given by

$$
\langle f(\xi), g(\xi)\rangle=\mathrm{E}[f(\xi) g(\xi)]=\int_{\Omega_{\xi}} f(\tau) g(\tau) \rho(\tau) \mathrm{d} \tau .
$$

Loosely speaking, PCE is applicable, because the above weighting functions $\rho(\cdot)$ of the polynomials from Table I correspond to the probability density functions of certain standard random variables.

PCE according to (6) is impractical due to the infinite sum. Consequently, only the first $L+1=\left(n_{\xi}+d\right) ! /\left(n_{\xi} ! d !\right)$ basis polynomials in $n_{\xi}$ variables of degree at most $d$ are considered, spanning a finite subspace of $\mathrm{L}^{2}\left(\Omega_{\xi}, \mathrm{F}_{\xi}, \mathrm{P}_{\xi}\right)$. This yields (with slight abuse of notation),

$$
z(\xi)=\sum_{l=0}^{L} z_{l} \phi_{l}(\xi)=\boldsymbol{z}^{\top} \boldsymbol{\phi}(\xi),
$$

where $\boldsymbol{z}=\left[z_{0}, \ldots, z_{L}\right]^{\top}$, and $\boldsymbol{\phi}(\xi)=\left[\phi_{0}(\xi), \ldots, \phi_{L}(\xi)\right]^{\top}$. Since the space $\mathrm{L}^{2}\left(\Omega_{\xi}, \mathrm{F}_{\xi}, \mathrm{P}_{\xi}\right)$ is a Hilbert space, the truncated PCE (8) - which is an element of the subspace spanned by the $L+1$ basis polynomials-is $\mathrm{L}^{2}$-optimal if the PCE coefficients satisfy

$$
z_{l}=\frac{\left\langle z(\xi), \phi_{l}(\xi)\right\rangle}{\left\langle\phi_{l}(\xi), \phi_{l}(\xi)\right\rangle}, \quad \forall j \in\{0, \ldots, L\},
$$

\footnotetext{
${ }^{3} \mathrm{~A}$ random variable is called a second-order random variable if it has finite variance. This is true for most physical processes. Furthermore, $\Omega_{\xi}$ is a countable event space, $\mathrm{F}_{\xi}$ is the $\sigma$-field of $\Omega_{\xi}$, and $\mathrm{P}_{\xi}$ is a probability measure. The triplet $\left(\Omega_{\xi}, \mathrm{F}_{\xi}, \mathrm{P}_{\xi}\right)$ is a probability space [12].
}

cf. [26, Section 3.3, Theorem 1]. If all PCE coefficients $z_{l}$ with $j \in\{0, \ldots, L\}$ are known, the PDF of $z(\xi)$ is easily obtained by sampling $\xi$. This is numerically straightforward, because by assumption $\xi$ is composed of standard random variables.

Remark 2 (Galerkin projection): Computing the PCE coefficients according to (9) is often called Galerkin projection, [12]. From an engineering perspective, this is equivalent to, e.g. finding Fourier coefficients, solving linear partial differential equations via separation of variables, or solving ordinary differential equations in modal form.

Remark 3 (Moments): Orthogonality of the basis polynomials allows to compute moments of random variables easily. For example, $\mathrm{E}[z(\xi)]=z_{0}$, and $\mathrm{E}\left[z(\xi)^{2}\right]=$ $\sum_{l=0}^{L} z_{l}^{2}\left\langle\phi_{l}(\xi), \phi_{l}(\xi)\right\rangle$.

\section{B. Stochastic AC Power Flow Equations Using PCE}

Section II introduced two different power flow formulations: polar and rectangular, cf. (2) and (3). The stochastic $\mathrm{AC}$ power flow problem can be solved with PCE and Galerkin-projection only for polynomial nonlinearities directly, i.e. rectangular power flow (3). ${ }^{4}$ Introduce (truncated) PCE according to (8) for all bus states $x_{i}$ from (3b),

$$
x_{i}(\xi):=\sum_{l=0}^{L} x_{i, l} \phi_{l}(\xi)=\boldsymbol{x}_{i} \phi(\xi) .
$$

Each bus is then described by $4(L+1)$ PCE coefficients $\boldsymbol{x}_{i}$,

$$
\boldsymbol{x}_{i}^{\top}=\left[\begin{array}{llll}
\boldsymbol{v}_{i}^{\mathrm{re}} & \boldsymbol{v}_{i}^{\mathrm{im}} & \boldsymbol{p}_{i} & \boldsymbol{q}_{i}
\end{array}\right] \in \mathbb{R}^{4 \times(L+1)} .
$$

In general, every quantity may be stochastic, i.e. described by its PDF. To obtain (nonlinear algebraic) equations for $2 N(L+1)$ PCE coefficients, we introduce the PCE according to (10) for rectangular power flow (3), and apply Galerkin projection. The resulting nonlinear algebraic equations for the PCE coefficients for active and reactive power are given in Table II. Note that PCE preserves the complexity of the original problem: the deterministic equations are quadratic, so is the Galerkin-projected system. It is the dimension of the problem that changes from $2 N$ to $2 N(L+1)$.

Remark 4 (PCE for polar power flow): Of course, PCE may be introduced for polar power flow (2), but orthogonality of the basis polynomials can then not be exploited via Galerkin projection. However, stochastic collocation is a viable option in this case [12, Ch. 7].

Remark 5 (Approximated stochastic polar power flow):

If the trigonometric functions in the polar power flow (2) were approximated by their Taylor series, the resulting approximated polar power flow equations would be polynomial. PCE can then be applied. However, even the first-order approximation, i.e. linearization, would result in a cubic power flow, because of the multiplication with $v_{i} v_{j}$. This would require quadruple scalar products of the basis polynomials. For rectangular power flow, only triple scalar products occur, cf. Table II.

\footnotetext{
${ }^{4}$ Directly here refers to the notion that the Galerkin-projected system has the same structure as the original stochastic formulation. This is true for PCE in combination with polynomial nonlinearities.
} 
TABLE II

GALERKIN PROJECTION FOR RECTANGULAR POWER FLOW (3).

\begin{tabular}{cl} 
Deterministic Eq. & Weighted PCE coefficient, $i \in \mathcal{N}, k \in\{0, \ldots, L\}$ \\
\hline (3c) & $\left\langle\phi_{k}, \phi_{k}\right\rangle p_{i, k}=\sum_{j=1}^{N} \sum_{l_{1}=0}^{L} \sum_{l_{2}=0}^{L}\left(g_{i j}\left(v_{i, l_{1}}^{\mathrm{re}} v_{j, l_{2}}^{\mathrm{re}}+v_{i, l_{1}}^{\mathrm{im}} v_{j, l_{2}}^{\mathrm{im}}\right)+b_{i j}\left(v_{i, l_{1}}^{\mathrm{im}} v_{j, l_{2}}^{\mathrm{re}}-v_{i, l_{1}}^{\mathrm{re}} v_{j, l_{2}}^{\mathrm{im}}\right)\right)\left\langle\phi_{l_{1}} \phi_{l_{2}}, \phi_{k}\right\rangle$ \\
(3d) & $\left\langle\phi_{k}, \phi_{k}\right\rangle q_{i, k}=\sum_{j=1}^{N} \sum_{l_{1}=0}^{L} \sum_{l_{2}=0}^{L}\left(g_{i j}\left(v_{i, l_{1}}^{\mathrm{im}} v_{j, l_{2}}^{\mathrm{re}}-v_{i, l_{1}}^{\mathrm{re}} v_{j, l_{2}}^{\mathrm{im}}\right)-b_{i j}\left(v_{i, l_{1}}^{\mathrm{re}} v_{j, l_{2}}^{\mathrm{re}}+v_{i, l_{1}}^{\mathrm{im}} v_{j, l_{2}}^{\mathrm{im}}\right)\right)\left\langle\phi_{l_{1}} \phi_{l_{2}}, \phi_{k}\right\rangle$ \\
\hline
\end{tabular}

\section{Stochastic Bus Specifications Using PCE}

The main purpose of stochastic power flow problems is to consider uncertain power load and/or uncertain generation explicitly. This information enters via PDFs at each bus, according to its bus specification, cf. (4). For PCE applied to rectangular power flow, the deterministic bus specifications (4) are extended easily for slack and PQ buses,

$$
\begin{gathered}
\text { Slack: }\left[\begin{array}{llll}
1 & 0 & 0 & 0 \\
0 & 1 & 0 & 0
\end{array}\right] \boldsymbol{x}_{i} \stackrel{!}{=}\left[\begin{array}{c}
\boldsymbol{v}^{\mathrm{ref} \top} \\
\mathbf{0}
\end{array}\right] \Leftrightarrow \boldsymbol{C}^{\mathrm{sl}} \boldsymbol{x}_{i}=\boldsymbol{c}_{i}^{\mathrm{sl}}, \\
\text { PQ: }\left[\begin{array}{llll}
0 & 0 & 1 & 0 \\
0 & 0 & 0 & 1
\end{array}\right] \boldsymbol{x}_{i} \stackrel{!}{=}\left[\begin{array}{l}
\boldsymbol{p}_{i}^{\mathrm{ref} \top} \\
\boldsymbol{q}_{i}^{\mathrm{ref} \top}
\end{array}\right] \Leftrightarrow \boldsymbol{C}^{\mathrm{pq}} \boldsymbol{x}_{i}=\boldsymbol{c}_{i}^{\mathrm{pq}}, \\
\text { PV: }\left[\begin{array}{llll}
0 & 0 & 1 & 0
\end{array}\right] \boldsymbol{x}_{i} \stackrel{!}{=}\left[\boldsymbol{p}_{i}^{\mathrm{ref} \top}\right] \Leftrightarrow \boldsymbol{C}^{\mathrm{pv}} \boldsymbol{x}_{i}=\boldsymbol{c}_{i}^{\mathrm{p}},
\end{gathered}
$$

where $\boldsymbol{x}_{i}$ contains all PCE coefficients, cf. (10), and the right-hand sides are the stacked reference PCE coefficients, specifying the bus uncertainty. Overall, $\left(2 N-N_{g}+1\right)(L+1)$ equality constraints are defined by (12). To cope with the remaining voltage constraints of the $N_{g}$ PV buses we assume the following:

Assumption 1 (Regulated Generators): The slack bus reference values for voltage magnitude and voltage angle, $v^{\text {ref }}$ and $\delta^{\text {ref }}=0$, are not subject to any uncertainty. Furthermore, the voltage $v_{i}^{\text {ref }}$ at each PV bus is regulated and thus not subject to any uncertainty, cf. (4). The corresponding vectors of PCE coefficients $\boldsymbol{v}^{\text {ref }}, \boldsymbol{\delta}^{\text {ref }}, \boldsymbol{v}_{i}^{\text {ref }} \in \mathbb{R}^{L+1}$ are non-zero only in their first, i.e. zero-order, entry.

From Assumption 1 it follows that voltage constraints for PV buses become nonlinear in (12), namely

$$
v_{i}(\xi)^{2}=\left(\boldsymbol{v}_{i}^{\mathrm{re} \top} \boldsymbol{\phi}(\xi)\right)^{2}+\left(\boldsymbol{v}_{i}^{\mathrm{im} \top} \boldsymbol{\phi}(\xi)\right)^{2} \stackrel{!}{=} v_{i, 0}^{2},
$$

where $i \in \mathcal{N}_{g}$. Of the $\left(\begin{array}{c}L+2 \\ L\end{array}\right)$ coefficients, the first one becomes $\left(v_{i, 0}^{\mathrm{re}}\right)^{2}+\left(v_{i, 0}^{\mathrm{im}}\right)^{2}=v_{i, 0}^{2}$, and the others equate to zero. Thus, for $L=2$ and Assumption 1, we have $4 N(L+1)$ equations for $4 N(L+1)$ unknowns: $2 N(L+1)$ equations from Galerkin projection, see Table II, and $2 N(L+1)$ equations from the bus specifications. For $L>2$ we have $\left(\begin{array}{c}L+2 \\ L\end{array}\right)-2(L+1)$ more equations than unknowns for each PV bus.

\section{Stochastic Optimal AC Power Flow}

Power demand and generation have to be given in form of bus specifications to solve deterministic as well as stochastic power flow problems. In optimal power flow problems, however, the power generation is not known a priori, rather computed such that a cost function is minimized, cf. (2) and (3). Using PCE as given in (10) stochastic (rectangular) optimal power flow can be formulated as follows:

$$
\begin{array}{ll}
\underset{\boldsymbol{x} \in \mathbb{R}^{4 N \times(L+1)}}{\operatorname{minimize}} & J(\boldsymbol{x}) \\
\text { subject to } & \text { PCE power flow, cf. Table II, } \\
& \boldsymbol{C}^{\mathrm{pq}} \boldsymbol{x}_{i}=\boldsymbol{c}_{i}^{\mathrm{pq}}, \quad \forall i \in \mathcal{N} \backslash \mathcal{N}_{g}, \\
& g(\boldsymbol{x}) \leq 0,
\end{array}
$$

where $\boldsymbol{x}=\left[\begin{array}{lll}\boldsymbol{x}_{1}^{\top} & \ldots & \boldsymbol{x}_{N}^{\top}\end{array}\right]^{\top} \in \mathbb{R}^{4 N \times(L+1)}$ comprises the PCE decision variables, cf. (11). The cost function $J$ : $\mathbb{R}^{4 N \times(L+1)} \rightarrow \mathbb{R}$ may represent expected values of active power generation, or expected values of line losses. However, it is also possible to penalize variances or other higherorder moments by using PCE coefficients, cf. Remark 3. Due to equality constraints (13b) and (13c), the number of degrees of freedom is reduced to $2 N_{g}(L+1)$. In general, the inequality constraints (13d) are nonlinear functions of the PCE coefficients $\boldsymbol{x}$. Similar to the cost function $J$, they may represent expected values of, e.g. active power generation limits. Of course, it is possible to consider higher-order moments, cf. Remark 3. Specifically, chance constraints may be incorporated to account for, e.g., transmission congestion in a probabilistic manner. Note that using PCE the stochastic optimal power flow problem is reformulated as a deterministic optimal power flow problem (13a) of larger dimension in the decision variables. In general, the stochastic optimal power flow problem described by (13a) is non-convex due to non-convexity of the constraints.

\section{IEEE 14 Bus EXAMPLE}

We demonstrate stochastic (optimal) AC power flow using PCE for the IEEE 14 bus example with 5 generators, $\mathcal{N}_{g}=$ $\{1,2,3,6,8\}$, see Figure 1. For line parameters and the deterministic values for PQ and PV buses see the Power Systems Test Case Archive. ${ }^{5}$ We subsume power generation and power demand under a single net power injection, e.g. active power for bus $i: p_{i}=p_{i}^{g}-p_{i}^{l}$, where $p_{i}^{g}>0$ is the generation and $p_{i}^{l}>0$ is the load. All units in p.u. refer to the base value $100 \mathrm{MVA}$. Uncertainty is specified via Beta distributions, which have a bounded support-a major advantage in modeling compared to Gaussians. The infinite

\footnotetext{
${ }^{5}$ Curated by University of Washington, Electrical Engineering, https://www.ee.washington.edu/research/pstca, visited March 11, 2016.
} 


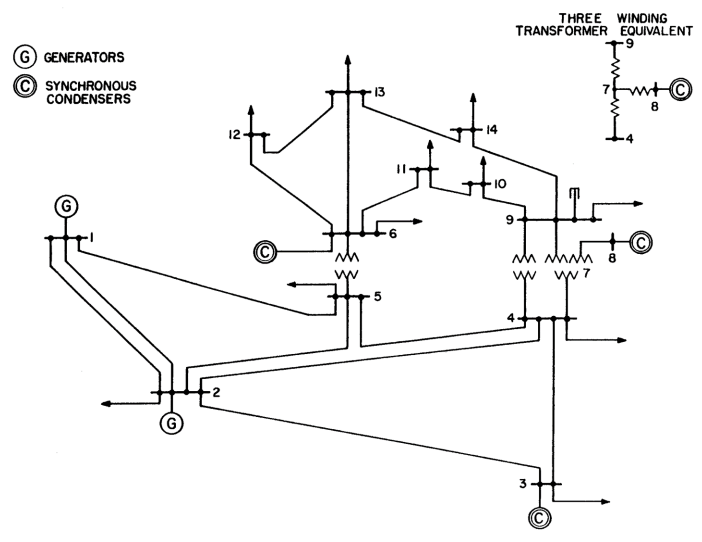

Fig. 1. IEEE 14 bus example with 5 generation buses, cf. Footnote 5.

support of Gaussian distributions may create difficulties in modeling the volatility of renewable generation units and/or power demands. As shown in [25], aggregated load profiles are more accurately modeled via Beta or Gamma distributions. Both can be handled by the PCE framework, cf. Table I, further motivating its use. According to Tables I and I, PCE is introduced using univariate Jacobi polynomials of order 2 for rectangular power flow, cf. Table II, resulting in $L=2$.

\section{A. Stochastic Power Flow}

First, we consider stochastic AC power flow using PCE. Uncertainty is assumed to enter via PDFs for active power demands at load buses 4, 9,11, and is specified as (symmetric) Beta distributions with shape parameters $a=b=3$. The corresponding PCE coefficients are

$$
\begin{aligned}
& \boldsymbol{p}_{4}^{\top}=[-0.4780,0.0239,0], \boldsymbol{q}_{4}^{\top}=[0.0390,0.0019,0], \\
& \boldsymbol{p}_{9}^{\top}=[-0.2950,0.0147,0], \boldsymbol{q}_{9}^{\top}=[-0.1660,0.0083,0], \\
& \boldsymbol{p}_{11}^{\top}=[-0.0350,0.0147,0], \boldsymbol{q}_{11}^{\top}=[-0.0180,0.0083,0] .
\end{aligned}
$$

Since we assume rectangular power flow, the PV bus constraints have to be formulated according to (12d). The PCE order is $L=2$. Thus, the total number of PCE coefficients amounts to $4 N(L+1)=168$. The system of nonlinear algebraic equations, defined by $2 N(L+1)$ equations from Table II and $2 N(L+1)$ bus specifications (12), is solved using Matlab. Exemplarily for the slack bus 1, the resulting PCE coefficients for active and reactive power are

$$
\begin{aligned}
& \boldsymbol{p}_{1}^{\top}=[2.4297,-0.0451,0.0001], \\
& \boldsymbol{q}_{1}^{\top}=[-0.1871,0.0047,0.0000] .
\end{aligned}
$$

The PCE histograms are obtained as follows: generate $m=1000$ samples for the standard Beta-distributed variable $\xi$ (with shape parameters from above, $a=b=3$ ), evaluate basis polynomials, then evaluate (8) for the respective PCE coefficients. Figure 2 shows the corresponding histograms, plotted against the benchmark Monte Carlo solution for the same number of $m=1000$ samples. Only the distributions of active and reactive power are shown in Figure 2, because the

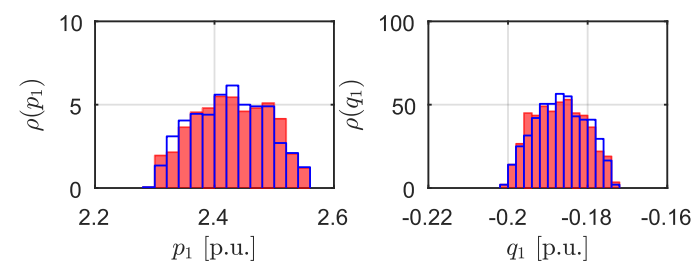

Fig. 2. Resulting PDFs of active and reactive power injection at slack bus 1 for stochastic power flow. Solid blue histograms in front denote histograms from sampled PCE coefficient solution (15a), red histograms in back denote Monte Carlo solution, each obtained for $m=1000$ samples.
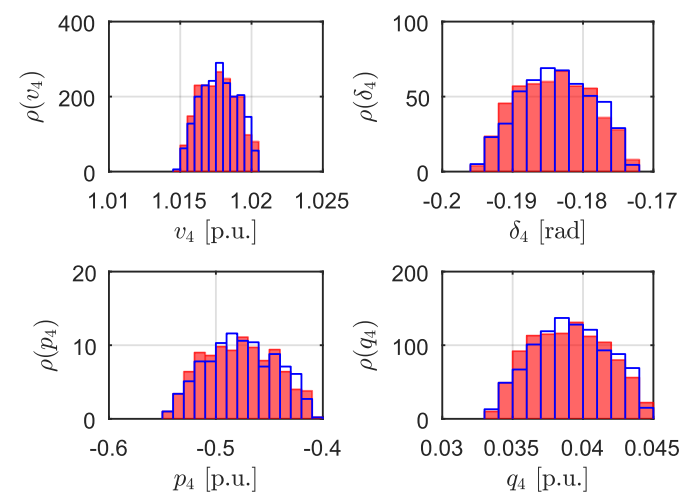

Fig. 3. Resulting PDFs of voltage magnitude and voltage angle of load bus 4 for stochastic power flow. PDFs obtained for given PCE coefficients $\boldsymbol{p}_{4}$, $\boldsymbol{q}_{4}$, cf. (14), and computed PCE coefficients $\boldsymbol{v}_{4}, \boldsymbol{\delta}_{4}$, cf. (15b). See caption of Figure 2 for color code and sample size.

voltage magnitude and its angle are assumed deterministic, cf. Assumption 1. Figure 3 shows the distributions of all four quantities for the load bus 4 . The PCE coefficients for voltage angle and voltage magnitude are computed as

$$
\begin{aligned}
\boldsymbol{v}_{4}^{\top} & =[1.0177,0.0010,0.0000], \\
\boldsymbol{\delta}_{4}^{\top} & =[-0.1840,0.0038,0.0000] .
\end{aligned}
$$

The active and reactive distributions follow from the initial PCE coefficients $\boldsymbol{p}_{4}, \boldsymbol{q}_{4}$, cf. (14). As can be seen, the resulting distributions remain (numerically) symmetrical. The numerical differences between the Monte Carlo solution and the PCE solution are negligible. However, already for $m=1000$ samples the differences in computational times are considerable: $855 \mathrm{~s}$ for Monte Carlo compared to $55 \mathrm{~s}$ for PCE. Note that for the polynomial order $L=2$, the influence of the PCE coefficients of order 2 is marginal, cf. (15), suggesting that orders $L>2$ yield no significant numerical benefits for this example.

\section{B. Stochastic Optimal Power Flow}

We now turn to the stochastic optimal AC power flow problem using PCE. Uncertainty is assumed to enter via PDFs for active power demands at load buses 4 and 5, and is specified as (nonsymmetric) Beta distributions with shape parameters $a=2, b=4$. The corresponding PCE coefficients 
are

$$
\begin{aligned}
& \boldsymbol{p}_{4}^{\top}=[-0.4780,0.0239,0], \boldsymbol{q}_{4}^{\top}=[0.0390,0.0019,0], \\
& \boldsymbol{p}_{5}^{\top}=[-0.076,0.0038,0], \boldsymbol{q}_{5}^{\top}=[-0.016,0.0008,0] .
\end{aligned}
$$

Of the $\left|\mathcal{N}_{g}\right|=5$ generators, the buses 3,6 , and 8 are synchronous condensers, see Figure 1. Their corresponding shafts rotate freely, such that they do not supply or demand any active power, i.e. $p_{i}(\xi) \equiv 0$ for all $i \in\{1,2,3\}$. Thus, we distinguish the set of active power generation buses as $\mathcal{N}_{g}^{\mathrm{p}}=\{1,2\}$ with $\left|\mathcal{N}_{g}^{\mathrm{p}}\right|=N_{g}^{\mathrm{p}}=2$. The cost function $J(\boldsymbol{x})$ represents the total active power generation cost associated with $\mathcal{N}_{g}^{\mathrm{p}}$, each assumed convex quadratic, i.e.

$$
\begin{aligned}
J(\mathrm{E}[x(\xi)]) & =\sum_{i \in \mathcal{N}_{g}^{\mathrm{p}}} a_{i}\left(\mathrm{E}\left[p_{i}(\xi)\right]+p_{i}^{l}\right)^{2}+b_{i}\left(\mathrm{E}\left[p_{i}(\xi)\right]+p_{i}^{l}\right) \\
& =\sum_{i \in \mathcal{N}_{g}^{\mathrm{p}}} a_{i}\left(p_{i, 0}+p_{i}^{l}\right)^{2}+b_{i}\left(p_{i, 0}+p_{i}^{l}\right) \\
& =\left(p_{0}+p^{l}\right)^{\top} A\left(p_{0}+p^{l}\right)+b^{\top}\left(p_{0}+p^{l}\right)
\end{aligned}
$$

where monetary costs are specified by $A=\operatorname{diag}\left(a_{1}\right.$, $\left.\ldots, a_{N_{g}^{\mathrm{p}}}\right) \in \mathbb{R}^{N_{g}^{\mathrm{p}} \times N_{g}^{\mathrm{p}}}$, and $b=\left[b_{1}, \ldots, b_{N_{g}^{\mathrm{p}}}\right]^{\top} \in \mathbb{R}^{N_{g}^{\mathrm{p}}}$. Also, $p_{0}=\left[p_{1,0}, p_{2,0}\right]^{\top} \in \mathbb{R}^{N_{g}^{\mathrm{p}}}$ contains the zero-order PCE coefficients of the synchronous generator buses, and $p^{l}=[0,0.217]^{\top} \in \mathbb{R}^{N_{g}^{\mathrm{p}}}$ contains the active power load demands of the respective generators. Recall Remark 3 for replacing the expected value with the zero-order PCE coefficient. The monetary costs are specified via

$$
\begin{aligned}
A & =\operatorname{diag}(430.3,2500) \$ / \text { h/p.u. }{ }^{2} \in \mathbb{R}^{N_{g}^{\mathrm{p}} \times N_{g}^{\mathrm{p}}}, \\
b & =[2000,2000]^{\top} \$ / \text { h/p.u. } \in \mathbb{R}^{N_{g}^{\mathrm{p}}} .
\end{aligned}
$$

There are $2 N(L+1)$ power flow equality constraints according to Table II, $2\left(N-N_{g}\right)(L+1)$ PQ bus equality constraints, cf. (12b), $N_{g}^{\mathrm{p}}(L+1)$ equality constraints for the active power PCE coefficients of the synchronous condensers, and additionally the slack bus constraints (12a) for all PCE coefficients except $v_{1,0}$. The inequality constraints are given in terms of expected values,

$$
\begin{aligned}
0.94 & \leq \mathrm{E}\left[v_{i}(\xi)\right]=v_{i, 0} \leq 1.06, & & \forall i \in \mathcal{N}, \\
p_{i, \min }-p_{i}^{l} \leq \mathrm{E}\left[p_{i}(\xi)\right] & =p_{i, 0} \leq p_{i, \max }-p_{i}^{l} & & \forall i \in \mathcal{N}_{g}^{\mathrm{p}}, \\
q_{i, \min }-q_{i}^{l} \leq \mathrm{E}\left[q_{i}(\xi)\right] & =q_{i, 0} \leq q_{i, \max }-q_{i}^{l} & & \forall i \in \mathcal{N}_{g}
\end{aligned}
$$

where $\mathcal{N}=\{1, \ldots, 14\}$. Due to the voltage inequality constraints the function $g(\boldsymbol{x})$ is nonlinear. The lower and upper limits for (re-)active power and the reactive power load demand of the generators are given by the following vectors

$$
\begin{aligned}
p_{\min } & =[0,0]^{\top}, \quad p_{\max }=[3.32,1.4]^{\top}, \\
q_{\min } & =[0,-0.4,0,-0.06,-0.06]^{\top}, \\
q_{\max } & =[0.1,0.5,0.4,0.24,0.24]^{\top}, \\
q^{l} & =[0,0.127,0.129,0.075,0]^{\top},
\end{aligned}
$$

where the entries follow the order of the entries in $\mathcal{N}_{g}^{p}$ and $\mathcal{N}_{g}$, respectively. The resulting optimization problem is solved using an interior-point method available via fmincon
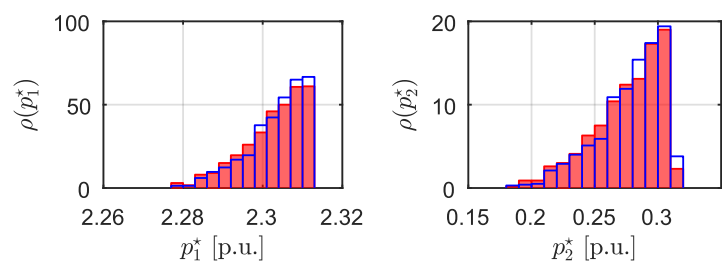

Fig. 4. Resulting PDFs of active power injections of generator buses 1 and 2 for stochastic optimal power flow for PCE coefficients (17a) and (17c). See caption of Figure 2 for color code and sample size.
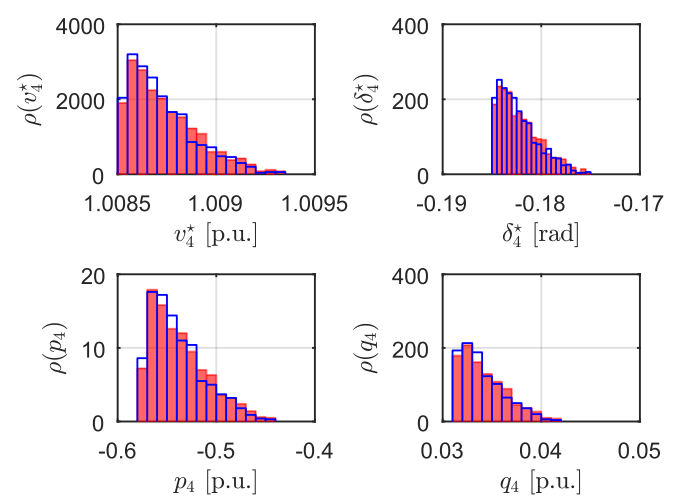

Fig. 5. Resulting PDFs of voltage magnitude and voltage angle of load bus 4 for stochastic optimal power flow. PDFs for active power and reactive power obtained from (16) as nonsymmetric Beta distributions. See caption of Figure 2 for color code and sample size.

in Matlab. The resulting PCE coefficients for active and reactive power at the generator buses are

$$
\begin{aligned}
\boldsymbol{p}_{1}^{\star} & =[2.2867,-0.0065,0.0000]^{\top}, \\
\boldsymbol{q}_{1}^{\star} & =[0.0000,0.0043,-0.0001]^{\top}, \\
\boldsymbol{p}_{2}^{\star} & =[0.2193,-0.0229,0.0000]^{\top}, \\
\boldsymbol{q}_{2}^{\star} & =[0.1655,-0.0041,0.0000]^{\top} .
\end{aligned}
$$

The PDFs of the active power injections for the generators are shown in Figure 4. They are plotted against the benchmark solution obtained from $m=1000$ Monte Carlo simulations for polar power flow. They are skewed to the right, i.e. towards higher supply. This behaviour is meaningful if compared the PDFs of (re-)active power demand of load bus 4, see Figure 5, which are skewed to the left, i.e. towards higher demand. These PDFs follow directly from the specified PCE coefficients (16). The PDFs for voltage magnitude and angle for load bus 4 follow the PDFs of the (re-)active power qualitatively. As observed in the stochastic power flow problem from Section IV-A, the influence of resulting PCE coefficients of order 2 is negligible, cf. (17). This suggests that a polynomial order $L=2$ is sufficient for this specific example. The optimal cost function resulting from (17) is $8171.96 \$ / \mathrm{h}$. It is in good agreement with the deterministic optimum of $8171.89 \$ / \mathrm{h}$, which is obtained from setting PCE coefficients of order one and higher of the generation buses to zero, and solving the corresponding problem. Whereas the numerical difference between the PCE 
and Monte Carlo solution is negligible, the computational effort is not $^{6}$ : it takes about $223 \mathrm{~s}$ to solve the optimization problem (13a), compared to a total of $2397 \mathrm{~s}$ for the entire Monte Carlo simulation. All problems are initiated with the same initial condition and $m=1000$ samples are considered.

\section{CONCLUSIONS AND OUTLOOK}

In the present contribution we discuss the use of polynomial chaos expansion for stochastic (optimal) AC power flow problems. PCE in conjunction with Galerkin projection is applicable to polynomial nonlinearities and thus to rectangular power flow. The result is a deterministic power flow problem of increased dimension but same mathematical structure. Compared to Monte Carlo approaches that require repeated sampling, PCE provides posterior distributions after a single run. Furthermore, PCE is applicable to arbitrary stochastic uncertainties described by probability density functions with finite variance. By means of the IEEE 14 bus system, we show that already a low order of polynomial approximation allows accurate computation of the posterior probability density functions.

Future work will consider the extension to other cost functions and chance constraints. Furthermore, we will investigate how to apply PCE to distributed solution schemes.

\section{REFERENCES}

[1] J. Grainger and W. Stevenson, Power System Analysis. McGraw-Hill Education, 1994.

[2] P. Kundur, Power System Stability and Control. McGraw-Hill Education, 1994.

[3] G. Hug-Glanzmann and G. Andersson, "Decentralized optimal power flow control for overlapping areas in power systems," Power Systems, IEEE Transactions on, vol. 24, no. 1, pp. 327-336, 2009.

[4] B. Kocuk, S. Dey, and X. Sun, "Inexactness of sdp relaxation and valid inequalities for optimal power flow," Power Systems, IEEE Transactions on, vol. 31, no. 1, pp. 642-651, 2016.

[5] Y. Taiyou and R. Lasseter, "Stochastic optimal power flow: Formulation and solution," in IEEE Power Engineering Society Summer Meeting, vol. 1, pp. 237-242, 2000.

[6] C. Hans, P. Sopasakis, A. Bemporad, J. Raisch, and C. ReinckeCollon, "Scenario-based model predictive operation control of islanded microgrids," in 54th IEEE Conference on Decision and Control (CDC), pp. 3272-3277, 2015.

[7] B. Borkowska, "Probabilistic load flow," IEEE Transactions on Power Apparatus and Systems, vol. PAS-93, pp. 752-759, May 1974.

[8] R. Allan, C. Grigg, and M. Al-Shakarchi, "Numerical techniques in probabilistic load flow problems," International Journal for Numerical Methods in Engineering, vol. 10, no. 4, pp. 853-860, 1976.

[9] N. Hatziargyriou, T. Karakatsanis, and M. Papadopoulos, "Probabilistic load flow in distribution systems containing dispersed wind power generation," IEEE Transactions on Power Systems, vol. 8, pp. 159165, Feb 1993.

[10] R. Allan, A. Leite da Silva, and R. Burchett, "Evaluation methods and accuracy in probabilistic load flow solutions," IEEE Transactions on Power Apparatus and Systems, vol. PAS-100, pp. 2539-2546, May 1981.

[11] P. Zhang and S. Lee, "Probabilistic load flow computation using the method of combined cumulants and Gram-Charlier expansion," IEEE Transactions on Power Systems, vol. 19, no. 1, pp. 676-682, 2004.

[12] D. Xiu, Numerical Methods for Stochastic Computations. Princeton University Press, 2010.

\footnotetext{
${ }^{6}$ All solutions were obtained via rapid prototyping using Matlab's symbolic toolbox, automatic code generation, and fmincon as solver. The absolute computation times can thus be drastically reduced for both scenarios. However, the relative difference between the computation times will not be greatly affected.
}

[13] H. Yu, C. Chung, K. Wong, H. Lee, and J. Zhang, "Probabilistic load flow evaluation with hybrid latin hypercube sampling and cholesky decomposition," IEEE Transactions on Power Systems, vol. 24, pp. 661667, May 2009.

[14] E. Rosenblueth, "Point estimates for probability moments," Proceedings of the National Academy of Sciences, vol. 72, no. 10, pp. 38123814, 1975.

[15] H. Hong, "An efficient point estimate method for probabilistic analysis," Reliability Engineering \& System Safety, vol. 59, no. 3, pp. 261267, 1998.

[16] M. Harr, "Probabilistic estimates for multivariate analyses," Applied Mathematical Modelling, vol. 13, no. 5, pp. 313-318, 1989.

[17] G. Verbić and C. Cañizares, "Probabilistic optimal power flow in electricity markets based on a two-point estimate method," IEEE Transactions on Power Systems, vol. 21, pp. 1883-1893, Nov 2006.

[18] J. Morales and J. Perez-Ruiz, "Point estimate schemes to solve the probabilistic power flow," IEEE Transactions on Power Systems, vol. 22, pp. 1594-1601, Nov 2007.

[19] S. Julier and J. Uhlmann, "New extension of the Kalman Filter to nonlinear systems," in Proceedings of Signal Processing, Sensor Fusion, and Target Recognition, vol. 3068, pp. 182-193, 1997.

[20] M. Aien, M. Fotuhi-Firuzabad, and F. Aminifar, "Probabilistic load flow in correlated uncertain environment using unscented transformation," IEEE Transactions on Power Systems, vol. 27, pp. 2233-2241, Nov 2012.

[21] O. Oke, D. Thomas, G. Asher, and L. de Menezes, "Probabilistic load flow for distribution systems with wind production using unscented transform method," in IEEE Innovative Smart Grid Technologies, pp. 1-7, Jan 2011.

[22] Z. Nagy and R. Braatz, "Distributional uncertainty analysis using power series and polynomial chaos expansions," Journal of Process Control, vol. 17, no. 3, pp. 229-240, 2007. Special Issue \{ADCHEM\} 2006 Symposium.

[23] A. Mesbah, S. Streif, R. Findeisen, and R. Braatz, "Stochastic nonlinear model predictive control with probabilistic constraints," in American Control Conference, pp. 2413-2419, June 2014.

[24] S. Lucia, P. Zometa, M. Kögel, and R. Findeisen, "Efficient stochastic model predictive control based on polynomial chaos expansions for embedded applications," in 54th Annual Conference on Decision and Control, pp. 3006-3012, Dec 2015.

[25] E. Carpaneto and G. Chicco, "Probabilistic characterisation of the aggregated residential load patterns," IET Generation, Transmission Distribution, vol. 2, pp. 373-382, May 2008.

[26] D. Luenberger, Optimization by Vector Space Methods. John Wiley \& Sons, Inc., 1969. 